\title{
Aufsätze
}

\section{UN-Kaufrecht oder Gemeinsames Europäisches Kaufrecht?}

\author{
Professor Dr. Phillip Hellwege, Augsburg*
}

\section{Einleitung}

Schon heute bietet das UN-Kaufrecht für einen Großteil Europas und darüber hinaus Regeln für grenzüberschreitende Kaufverträge an, ist es doch in fast allen Mitgliedstaaten der Europäischen Union sowie in allen ihren Beitrittskandidaten in Kraft getreten. Außen vor bleiben nur das Vereinigte Königreich, Irland, Malta und Portugal. Doch schon bald könnte das UN-Kaufrecht von einem Gemeinsamen Europäischen Kaufrecht Konkurrenz bekommen. Einen Vorschlag für eine Verordnung über ein solches Kaufrecht hat die Europäische Kommission am 11.10. 2011 vorgelegt. ${ }^{1}$ Nach Inkrafttreten eines Gemeinsamen Europäischen Kaufrechts wird sich den Marktteilnehmern in Europa die Frage stellen, ob sie dieses, das UN-Kaufrecht oder allein ein nationales Recht zur Anwendung kommen lassen wollen. ${ }^{2}$ Ziel des vorliegenden Beitrages ist es, Erwägungen herauszuarbeiten, von denen sich Marktteilnehmer bei ihrer Entscheidung zwischen dem UN-Kaufrecht und dem Gemeinsamen Europäischen Kaufrecht leiten lassen werden, soweit denn beide Instrumente auch an- bzw. verwendbar sind. ${ }^{3}$

Dafür müssen beide Instrumente miteinander verglichen werden. ${ }^{4}$ Marktteilnehmer werden dabei vor allem mit vier Fragen an die Instrumente herantreten: ${ }^{5}$ Erstens wird sie interessieren, welches Regelwerk höhere Transaktionskosten verursacht und mit welchem umgekehrt solchen Kosten eingespart werden können. Dass es genau dieser Punkt ist, an dem sich das Gemeinsame Europäische Kaufrecht messen lassen will, wird aus Art. 1(2) DCESL-VO deutlich: ${ }^{6}$

„Diese Verordnung ermöglicht es Unternehmern, sich bei allen ihren grenzübergreifenden Geschäften auf gemeinsame Vorschriften zu stützen und dieselben Vertragsbestimmungen zu verwenden, und hilft so, unnötige Kosten zu sparen und gleichzeitig ein hohes Maß an Rechtssicherheit herzustellen.“
* Der Beitrag schließt unmittelbar an den Beitrag „Die Geltungsbereiche des UN-Kaufrechts und des Gemeinsamen Europäischen Kaufrechts im Vergleich“ im letzten Heft der IHR (IHR 5/2012) an. Beide Beiträge zusammen basieren auf einem Vortrag, den der Autor am 12.4.2012 beim gemeinsam von den juristischen Fakultäten der Universitäten Ankara und Augsburg veranstalteten 2. Deutsch-Türkischen Rechtssymposium in Ankara gehalten hat. Das Manuskript wurde am 11.4.2012 abgeschlossen. Danach erschienene Literatur wurde für diese veröffentlichte Version noch berücksichtigt, sofern sie den Autoren bis zum 11.5.2012 erreicht hat.

1 Vorschlag für eine Verordnung des Europäischen Parlaments und des Rates über ein Gemeinsames Europäisches Kaufrecht, 11.10.2011, KOM (2011) 635 endg. Abgedruckt in: Schulze/Zimmermann (Hrsg.), Europäisches Privatrecht. Basistexte, 4. Aufl. 2012, S. 43-105.

2 Vgl. bereits Lando, in: Remien/Herrler/Limmer (Hrsg.), Gemeinsames Europäisches Kaufrecht für die EU?, 2012, S. 15 ff.

3 Vgl. zu den Schnittmengen im Geltungsbereich beider Instrumente meinen Beitrag im letzten Heft der IHR (IHR 5/2012).

4 Vgl. bereits Lando (o. Fn. 2), S. 15 ff.; Mankowski, IHR 2012, 47 ff. UNKaufrecht und die kaufrechtlichen Vorschriften des DCFR haben bereits verglichen: Huber, in: Festschr. f. Ingeborg Schwenzer, Bd. I, 2011, S. 807-826; Kruisinga, ERPL 2011, 907-919.

5 Unberücksichtigt bleiben generelle Erwägungen zur Qualität des Verordnungsvorschlages oder zu Kompetenzfragen: vgl. hierzu Remien, in: Remien/Herrler/Limmer (o. Fn. 2), S. 3 ff.; Eidenmüller/Jansen/Kieninger/ Wagner/Zimmermann, JZ 2012, 270, 269-289; Zimmermann, JBl 2012, 8 ff.; Ackermann, in: Remien/Herrler/Limmer (o. Fn. 2), S. 57 ff.; Grigoleit, in: Remien/Herrler/Limmer (o. Fn. 2), S. 75 ff.; Martens, AcP 211 (2011), 845-885; Feltkamp/Vanbossele, ERPL 2011, 873-905; Müller, GPR 2012, 11-20; Roth, EWS 2012, 12-21; Riesenhuber, EWS 2012, 6-12; Tamm, VuR 2012, 3-12; Basedow, EuZW 2012, 1-2; Staudenmayer, NJW 2011, 3495 f.; Kuipers, ERPL 2011, 545-564. Vgl. außerdem bereits Basedow, in: Festschr. f. Franz Jürgen Säcker, 2011, S. 29-44. Eine erste ausführliche Analyse findet sich in den Beiträgen in: Schmidt-Kessel (Hrsg.), Ein einheitliches europäisches Kaufrecht?, 2012; Schulte-Nölke/Zoll/Jansen/Schulze (Hrsg.), Der Entwurf für ein optionales europäisches Kaufrecht, 2012; Wendehorst/Zöchling-Jud (Hrsg.), Am Vorabend eines Gemeinsamen Europäischen Kaufrechts, 2012.

6 Siehe auch KOM (2011) 635 endg., S. 2 ff. der Begründung und Erwägungsgründe $2 \mathrm{ff}$. DCESL-VO. Vgl. hierzu Eidenmüller/Jansen/Kieninger/Wagner/Zimmermann, JZ 2012, 270, 286; Staudenmayer, NJW 2011, 3492; Ackermann (o. Fn. 5), S. 52 ff.; Grigoleit (o. Fn. 5), S. 70; Mankowski, IHR 2012, 46 . 
Die Marktteilnehmer werden zweitens wissen wollen, ob beide Instrumente gleichermaßen auf die besonderen Bedürfnisse des grenzüberschreitenden Verkehrs zugeschnitten sind. Sie werden drittens fragen, ob sie als Käufer oder Verkäufer durch eines der Regelwerke rechtlich besser gestellt werden als durch das andere. Schließlich wird sich Ihnen die Frage stellen, ob das Gemeinsame Europäische Kaufrecht in denjenigen Mitgliedstaaten der Europäischen Union auf Akzeptanz stoßen wird, die nicht Vertragsstaaten des UN-Kaufrechts sind. Denn einen wirklichen Mehrwert wird das Gemeinsame Europäische Kaufrecht gegenüber dem UN-Kaufrecht vor allem dann bringen, wenn es Marktteilnehmer etwa aus Großbritannien überzeugen wird und sie damit in dessen Verwendung einwilligen werden.

\section{Der Geltungsbereich beider Regelwerke}

Transaktionskostenrelevante Unterschiede zwischen beiden Regelwerken betreffen zunächst den Geltungsbereich beider Instrumente. Ist ein Marktteilnehmer mehreren Rechten oder Regelwerken ausgesetzt, so generiert dies Transaktionskosten: AGB müssen an die verschiedenen rechtlichen Rahmenbedingungen angepasst ${ }^{7}$ sowie die unterschiedlichen Bestimmungen bei der Vertragsabwicklung und den dabei auftauchenden Problemen beachtet werden.

Der räumliche Geltungsbereich des Gemeinsamen Europäischen Kaufrechts bietet prima facie Vorteile für diejenigen Marktteilnehmer, die ihren gewöhnlichen Aufenthalt in einem Mitgliedstaat der Europäischen Union haben und nicht nur mit solchen Geschäftspartnern in Kontakt stehen, die aus Vertragsstaaten des UN-Kaufrechts kommen. Denn für Verträge, die nicht in den Anwendungsbereich des Art. 1(1)(a) CISG fallen, müssen sie sich auf andere Rechte einlassen, sofern nicht der Weg über Art. 1(1)(b) CISG gegangen wird. Der Verordnungsvorschlag ermöglicht es dagegen Marktteilnehmern aus einem Mitgliedstaat, nur zum Gemeinsamen Europäischen Kaufrecht zu kontrahieren. Dass der Vertragspartner seinen gewöhnlichen Aufenthalt möglicherweise nicht in einem Mitgliedstaat hat, ist nach Art. 4 DCESL-VO unschädlich. ${ }^{8}$ Voraussetzung ist freilich, dass sich der Vertragspartner auf die Verwendung des Gemeinsamen Europäischen Kaufrechts nach Artt. 3 und 8 DCESL-VO einlässt und dass das Recht eines Mitgliedstaates kollisionsrechtlich einschlägig ist. ${ }^{9}$ Die letztgenannte Voraussetzung, dass das Recht eines Mitgliedstaates kollisionsrechtlich anwendbar sein muss, deutet aber bereits darauf hin, dass der transaktionsrelevante Vorteil des Gemeinsamen Europäischen Kaufrechts geringer ist, als auf den ersten Blick gedacht: Sind die Parteien im Sinne der Vorschaltlösung dazu gezwungen, das Recht eines Mitgliedstaates zu wählen, um sodann das Gemeinsame Europäische Kaufrecht wählen zu können, dann könnten sie auch nach Art. 1 (1)(b) CISG das Recht eines Vertragsstaates wählen und so das UN-Kaufrecht zur Anwendung zu bringen. ${ }^{10}$

Als Hindernis für den Erfolg des Gemeinsamen Europäischen Kaufrechts mag sich zudem eine Verwendbarkeitseinschränkung erweisen: ${ }^{11}$ Für einen Marktteilnehmer, der seinen gewöhnlichen Aufenthalt nicht in einem Mitgliedstaat hat, bedeutet es eine Erhöhung seiner Transaktionskosten, wenn er nur bei einem Vertragsschluss mit Vertragspartnern, die ihren gewöhnlichen
Aufenthalt in einem Mitgliedstaat haben, das Gemeinsame Europäische Kaufrecht wählen darf, für alle anderen Verträge aber auf das UN-Kaufrecht oder ein nationales Kaufrecht zurückgreifen muss. Für solche Vertragspartner ist das Gemeinsame Europäische Kaufrecht keine Alternative, sondern nur ein zusätzliches Kaufrechtsregime. Ein solcher Marktteilnehmer wird sich folglich dem Wunsch auf Verwendung des Gemeinsamen Europäischen Kaufrechts verschließen wollen. Das würde wiederum bedeuten, dass auch ein Marktteilnehmer mit gewöhnlichem Aufenthalt in einem Mitgliedstaat das Gemeinsame Europäische Kaufrecht nicht allen seinen Kaufverträgen zugrunde legen kann. Wiederum würde gelten, dass das Gemeinsame Europäische Kaufrecht keine Alternative, sondern nur ein zusätzliches Kaufrechtsregime wäre, das damit auch zusätzliche Kosten verursachen würde. Wäre das Gemeinsame Europäische Kaufrecht auch dann verwendbar, wenn keine der Parteien ihren gewöhnlichen Aufenthalt in einem Mitgliedstaat hat, solange sie nur zusätzlich zum Gemeinsamen Europäischen Kaufrecht auch das Recht eines Mitgliedstaates wählten, könnte dies nach alldem dessen Erfolg steigern. Dafür müsste Art. 4(2) und (3) DCESL-VO entsprechend geändert werden. ${ }^{12}$

Die Attraktivität des Gemeinsamen Europäischen Kaufrechts würde zudem noch gesteigert werden, wenn der Mitgliedstaat, in dem der Marktteilnehmer seinen gewöhnlichen Aufenthalt i.S.d. Art. 4 DCESL-VO hat, von der Option des Art. 13(a) DCESL-VO Gebrauch macht, so dass das Gemeinsame Kaufrecht auch für rein interne Sachverhalte verwendet werden darf. ${ }^{13}$ Der Marktteilnehmer könnte dann seine internationalen Warenkaufverträge ebenso wie seine rein internen Geschäfte unter der Geltung eines einheitlichen Regelwerkes abwickeln.

Freilich könnten die so ersparten Transaktionskosten auf anderer Seite wieder anfallen. Macht kein Mitgliedstaat von der Option nach Art. 13(b) DCESL-VO Gebrauch oder nutzen sie nur solche Mitgliedstaaten, die als Rahmenrechtsordnung für die Anwendung des Gemeinsamen Europäischen Kaufrechts wenig attraktiv sind, würde es zu einer Zweiteilung kommen: ${ }^{14}$ Bei Verträgen zwischen Unternehmern wäre das Gemeinsame Europäische Kaufrecht grundsätzlich nicht verwendbar. Nur bei Verträgen zwischen einem Unternehmer und einem kleineren und mittleren Unternehmen könnte es gewählt werden. Ein Marktteilnehmer, der sowohl mit kleinen und mittleren Unternehmen

$7 \mathrm{Zu}$ diesen Kosten Moser, in: Remien/Herrler/Limmer (o. Fn. 2), S. 8; Haug, K\&R 2012, 1; Graf von Westphalen, ZIP 2011, 1990.

8 Vgl. meinen Beitrag im letzten Heft der IHR (IHR 5/2012) unter Gliederungspunkt IV.

9 Vgl. hierzu meinen Beitrag im letzten Heft der IHR (IHR 5/2012) unter Gliederungspunkt VII.

10 Vgl. nur Ferrari, in: Schlechtriem/Schwenzer, 5. Aufl. 2008, Art. 1 Rn. 72.

11 Kritisch hierzu bereits mein Beitrag im letzten Heft der IHR (IHR 5/2012) unter Gliederungspunkt VII.

12 Dafür spricht sich wohl auch Schmidt-Kessel, in: ders. (o. Fn. 5), S. 38 f., aus.

13 Befürwortend auch von Westphalen, ZIP 2011, 1985; Herresthal, ZIP 2011, 1348; Leible, EuZW 2011, 809-810; ders., in: Remien/Herrler/Limmer (o. Fn. 2), S. 33; Lando (o. Fn. 2), S. 20; Ackermann (o. Fn. 5), S. 55; Perner, in: Wendehorst/Zöchling-Jud (o. Fn. 5), S. 24.

14 Vgl. auch die Kritik von Pfeiffer in: Remien/Herrler/Limmer (o. Fn. 2), S. 38 f.; Ernst, in: Remien/Herrler/Limmer (o. Fn. 2), S. 102; Zimmermann, JBl 2012, 18; Herresthal, ZIP 2011, 1348; Schmidt-Kessel, in: ders. (o. Fn. 5), S. 8 . 
als auch mit anderen Unternehmern grenzübergreifende Warenkaufverträge eingeht, sähe sich zwei Regelwerken ausgesetzt. Freilich würde es für kleinere und mittlere Unternehmen Sinn machen, das Gemeinsame Europäische Kaufrecht zu verwenden. Sie könnten es allen ihren Warenkaufverträgen mit anderen Unternehmern zugrunde legen, denn Art. 7(1)(2) DCESL-VO lässt es genügen, dass nur ein Vertragspartner ein kleines oder mittleres Unternehmen ist, und ohne Bedeutung ist, ob es sich dabei um den Käufer oder Verkäufer handelte. Hier ist am Ende die Verhandlungsstärke entscheidend: Das kleine oder mittlere Unternehmen müsste in der stärkeren Verhandlungsposition sein, um ein Unternehmen, das nicht ein Unternehmen i.S.d. Art. 7(2) DCESL-VO ist, gegen dessen ausdrücklichen Wunsch zur Verwendung des Gemeinsamen Europäischen Kaufrechts zu drängen. ${ }^{15}$

Transaktionskostenrelevant ist schließlich folgendes: Der Verordnungsvorschlag muss so verstanden werden, dass er auch Verträge zwischen Unternehmern als Verkäufer und Personen, die weder Unternehmer noch Verbraucher sind, als Käufer erfasst. ${ }^{16}$ Zwar werden solche Verträge weder in den Erwägungsgründen noch in der Begründung als Fallkonstellationen genannt, für die der Verordnungsentwurf ausgearbeitet worden ist. Sogar sprechen die Begründung und die Erwägungsgründe als Regelungsgegenstand explizit nur von Verträgen zwischen Verbrauchern und Unternehmern einerseits und Verträgen unter Unternehmern andererseits. ${ }^{17}$ Verträge mit einem Unternehmer auf Verkäuferseite und einer Person, die weder Verbraucher noch Unternehmer ist, auf Käuferseite werden auch im Kaufrechtsentwurf selbst konsequent ignoriert. ${ }^{18}$ Indes verlangt Art. 7(1) DCESL-VO nur, dass der Verkäufer ein Unternehmer ist. Nach dem allein maßgeblichen Art. 7(1) DCESL-VO sollte das Gemeinsame Europäische Kaufrecht also auch für Verträge zwischen einem Unternehmer als Verkäufer und etwa einem Idealverein als Käufer anwendbar sein. Dafür spricht weiterhin Art. 1 (2) DCESL-VO, der als Ziel des Verordnungsvorschlages bestimmt, dass die Verordnung „es Unternehmen [ermöglicht], sich bei allen ihren grenzübergreifenden Geschäften auf gemeinsame Vorschriften zu stützen und diesselben Vertragsbestimmungen zu verwenden“. Verträge mit Käufern, die weder Verbraucher noch Unternehmer sind, vom Geltungsbereich des Gemeinsamen Europäischen Kaufrechts auszuschließen, wäre mit diesem Ziel nicht vereinbar. Und die Ausklammerung solcher Verträge würde schließlich auch schlicht keinen Sinn machen, denn dann würden solche Verträge entweder enorme Transaktionskosten verursachen, wenn ein Unternehmer für alle anderen Verträge auf das Gemeinsame Europäische Kaufrecht zurückgreifen darf und nur für solche Verträge auf nationales Recht zurückfallen muss. Oder Unternehmer würden, wenn sie für alle anderen Fälle dennoch das Gemeinsame Europäische Kaufrecht verwenden, aber diese Kosten vermeiden wollen, auf Vertragsschlüsse mit solchen Personen verzichten.

Wir können mithin festhalten: In Mitgliedstaaten, die von den Optionen des Art. 13(a) DCESL-VO Gebrauch machen werden, kann die Verwendung des Gemeinsamen Europäischen Kaufrechts zu einer Verringerung von Transaktionskosten im Vergleich zur Verwendung des UN-Kaufrechts führen. Umgekehrt wird die Verwendung des Gemeinsamen Europäischen Kaufrechts zu höheren Transaktionskosten führen, wenn kein oder kein als Rah- menrechtsordnung attraktiver Mitgliedstaat von der Option des Art.13(b) DCESL-VO Gebrauch macht. Schließlich kann die Einschränkung des Art. 4(2) DCESL-VO dazu führen, dass die Verwendung des Gemeinsamen Europäischen Kaufrechts zu zusätzlichen Transaktionskosten führt, nämlich dann, wenn sich Marktteilnehmer aus Nichtmitgliedstaaten nicht auf das Gemeinsame Europäische Kaufrecht einlassen werden, weil sie es nicht für ihre übrigen Kaufverträge verwenden können.

\section{Die erfassten Regelungsmaterien beider Regelwerke}

Einen Vorteil bringt das Gemeinsame Europäische Kaufrecht gegenüber dem UN-Kaufrecht, wenn wir die erfassten Regelungsmaterien betrachten: Nach Art. 4 CISG regelt das UN-Kaufrecht „ausschließlich den Abschluss des Kaufvertrages und die aus ihm erwachsenden Rechte und Pflichten des Verkäufers und des Käufers“. Fragen der Gültigkeit von Verträgen und einzelner Vertragsbestimmungen sind ausdrücklich nicht vom UN-Kaufrecht erfasst (sogenannte externe Lücken). Besonders misslich, weil praxisrelevant, ist dabei, dass das UN-Kaufrecht keine Bestimmungen zur Inhaltskontrolle missbräuchlicher Klauseln, insbesondere von Allgemeinen Geschäftsbedingungen, enthält. ${ }^{19}$ Doch selbst die vom Übereinkommen geregelten Materien sind nicht abschließend erfasst (interne Lücken). Die Identifizierung von Lücken ist ebenso komplex wie ihre Schließung. Für externe Lücken ist immer, für interne Lücken nach Art. 7(2) CISG nur subsidiär auf das kollisionsrechtlich einschlägige nationale Recht abzustellen. ${ }^{20}$

Die Zahl der externen Lücken wurde im Gemeinsamen Europäischen Kaufrecht verringert. ${ }^{21}$ Insbesondere enthält das Gemeinsame Europäische Kaufrecht Bestimmungen zur Inhaltskontrolle missbräuchlicher Klauseln. ${ }^{22}$ Insoweit erübrigt sich der Rückgriff auf das nach den Regeln des Internationalen Privatrechts einschlägige Recht. Freilich weist auch das Gemeinsame Europäische Kaufrecht externe Lücken auf. So enthält es keine Bestimmungen zu gesetzes- oder sittenwidrigen Verträgen. Auch Fragen der Geschäftsfähigkeit sind nicht geregelt. Besonders misslich ist, dass das Gemeinsame Europäische Kaufrecht keine Regelungen zur Stellvertretung enthält, führt der Verord-

15 Vgl. hierzu kritisch Lando (o. Fn. 2), S. 19; Zimmermann, JBl 2012, 17 f.

16 Siehe hierzu meinen Beitrag im letzten Heft der IHR (IHR 5/2012) unter Gliederungspunkt III.

17 Siehe KOM (2011) 635 endg., S. 2 f., 13 der Begründung; Erwägungsgrund 21 DCESL-VO. Siehe außerdem Schulte-Nölke, in: Schulze/Stuyck (Hrsg.), Towards a European Contract Law, 2011, S. 41.

18 Siehe meinen Beitrag im letzten Heft der IHR (IHR 5/2012) unter Gliederungspunkt IV und unten den Text zu Fn. 69 und Fn. 84.

19 Hierzu Ferrari, in: Schlechtriem/Schwenzer (o. Fn. 10), Art. 4 Rn. 20; Staudinger/Magnus, CISG, 2005, Art. 4 Rn. 25 f.; Piltz, Internationales Kaufrecht, 2. Aufl. 2008, Rn. 3-91.

20 Paal, ZVglRWiss 110 (2011), 64-88; Ferrari, in: Schlechtriem/Schwenzer (o. Fn. 10), Art. 7 Rn. 43 ff.

21 So auch Perner (o. Fn. 13), S. 28. Zur Unterscheidung von internen und externen Lücken im Verordnungsvorschlag Gsell, in: Remien/Herrler/ Limmer (o. Fn. 2), S. 147 ff. Zur Schließung externer Lücken ebd., S. 154 ff.; KOM (2011) 635 endg., S. 7 der Begründung; Erwägungsgrund 27 DCESL-VO.

22 Dazu noch unten unter Gliederungspunkt VII. 
nungsentwurf doch aus, dass im Gemeinsamen Europäischen Kaufrecht alle Fragen beantwortet werden sollen, „die während des Lebenszyklus von Verträgen [...] von praktischer Bedeutung sind“. ${ }^{23}$ Dass unter dieser erklärten Zielsetzung die praktisch wichtige Frage der Stellvertretung nicht hätte ausgeklammert werden dürfen, ist bereits festgestellt worden. ${ }^{24}$ Unverständlich ist auch, dass bei einem Regelwerk, das in erster Linie für grenzüberschreitende Verträge geschaffen worden ist, Regeln zur Bestimmung der Vertragssprache fehlen ${ }^{25}$ und solche Regeln damit, folgt man der gerade zitierten Begründung, von den Verfassern wohl als praktisch unbedeutend angesehen werden. Trotz dieser Mängel bleibt indes positiv festzuhalten, dass die Zahl der externen Lücken im Vergleich zum UN-Kaufrecht geringer ausfällt.

Was interne Lücken angeht, so weicht Art. 4(2) DCESL entscheidend von Art. 7(2) CISG ab: ${ }^{26}$ Interne Lücken sind allein aus dem Gemeinsamen Europäischen Kaufrecht heraus und gerade ohne subsidiären Rückgriff auf das kollisionsrechtlich einschlägige nationale Recht zu schließen.

Nach alledem reduziert beides, sowohl die Verringerung der Anzahl externer Lücken wie auch die Bestimmung, dass interne Lücken ohne Rückgriff auf jedes nationale Recht zu schließen sind, Transaktionskosten.

\section{Gerichtliche Auslegung}

Ein weiterer Vorteil des Gemeinsamen Europäischen Kaufrechts gegenüber dem UN-Kaufrecht ist, dass dessen einheitliche Anwendung langfristig durch den EuGH gewährleistet werden könnte. ${ }^{27}$ Auch das wirkt sich transaktionskostensenkend aus, erübrigt sich doch so die Anpassung etwa von Klauseln an eine divergierende nationale Rechtsprechung. Freilich ist bereits festgestellt worden, dass der EuGH dieser Aufgabe derzeit nicht gewappnet ist. ${ }^{28}$

\section{Schadensersatzpflicht bei Verstoß gegen das Gebot von Treu und Glauben}

Wenden wir uns nun den einzelnen Kapiteln, Abschnitten und Artikeln des Gemeinsamen Europäischen Kaufrechts zu. Im Sinne der oben herausgearbeiteten Zielsetzung dieses Beitrages, steht nicht die dogmatische Kohärenz der Bestimmungen im Vordergrund. Auch die Tatsache, dass eine Bestimmung aus rechtsvergleichender oder aus deutscher Perspektive ungewöhnlich ist, ist für unsere Zwecke nicht entscheidend. Vielmehr sind diese Erkenntnisse nur von Bedeutung, soweit sie auf einen der oben genannten vier Bewertungsmaßstäbe Einfluss haben.

Eine ungewöhnlich scheinende Vorschrift findet sich in Art. 2 DCESL:

„1. Jede Partei hat die Pflicht, im Einklang mit dem Gebot von Treu und Glauben und des redlichen Geschäftsverkehrs zu handeln.

2. Verletzt eine Partei diese Pflicht, so kann sie das von der Ausübung oder Geltendmachung von Rechten, Abhilfen oder Einwänden, die ihr sonst zugestanden hätten, ausschließen, oder es kann sie für jeden Verlust, der der anderen Partei dadurch entsteht, haftbar machen.
3. Die Parteien dürfen die Anwendung dieses Artikels nicht ausschließen, davon abweichen oder dessen Wirkungen abändern."

Das Gebot von Treu und Glauben ist dem deutschen Auge vertraut. Ungewöhnlich ist aus deutscher Sicht, dass es sich nach Art. 2(1) DCESL um eine Rechtspflicht handelt, deren Verstoß nach Art. 2(2) DCESL zum Schadensersatz verpflichtet. Freilich ist die Bestimmung für den deutschen Betrachter nur auf den ersten Blick fremd. Denn auch nach deutschem Recht können sich aus $\$ 242$ BGB Nebenpflichten ergeben. ${ }^{29}$ Dass sich insbesondere Schutzpflichten aus dem Gebot von Treu und Glauben herleiten lassen, wird in $\$ 241$ II BGB ausdrücklich anerkannt. ${ }^{30}$ Der Verstoß gegen solche Pflichten kann zu einer Schadensersatzpflicht nach $\$ 280$ I BGB führen. ${ }^{31}$

Gleiches gilt für das UN-Kaufrecht. Obwohl es ein allgemeines Gebot von Treu und Glauben gerade nicht anerkannt hat, ${ }^{32}$ ist doch selbstverständlich, dass Nebenpflichten aus dem UNKaufrecht und aus den ihm unterfallenden Verträgen im Wege der Auslegung hergeleitet werden können und dass ein Verstoß gegen eine auf solche Weise begründete Nebenpflicht zu einer Schadensersatzpflicht führen kann. ${ }^{33}$

Man könnte also meinen, dass Art. 2 DCESL etwas Selbstverständliches enthält. Dennoch geht er über das hinaus, was das deutsche Recht und das UN-Kaufrecht anerkennen: Nach deutschem Recht führt nicht jeder Verstoß gegen Treu und Glauben zu einer Schadensersatzpflicht. Rechtsfolge kann vielmehr auch sein, dass einer Rechtsausübung Schranken gesetzt werden. ${ }^{34}$ Das Gebot von Treu und Glauben muss also zunächst eine Nebenpflicht begründen. Für das UN-Kaufrecht gilt ähnliches: Zunächst muss im Wege der Vertragsauslegung eine Nebenpflicht begründet werden. ${ }^{35}$ Art. 2 DCESL erweckt dagegen den Eindruck, dass jeder Verstoß gegen Treu und Glauben unmittelbar zu einer Schadensersatzpflicht führt. Zwar könnte man einwen-

23 Erwägungsgrund 26 DCESL-VO.

24 Siehe Eidenmüller/Jansen/Kieninger/Wagner/Zimmermann, JZ 2012, 271; Zimmermann, JBl 2012, 9-10; Grigoleit (o. Fn. 5), S. 75. A. A. Moser (o. Fn. 7), S. 12, die die Stellvertretung bei grenzüberschreitenden Verträgen für „weniger problematisch“ erachtet.

25 Siehe Erwägungsgrund 27 DCESL-VO.

26 Siehe auch Erwägungsgrund 29 DCESL-VO. Hierzu ausführlich Gsell (o. Fn. 21), S. 149 ff. Vgl. außerdem Haug, K\&R 2012, 1 f.

27 So auch Mankowski, IHR 2012, 47.

28 So von Eidenmüller/Jansen/Kieninger/Wagner/Zimmermann, JZ 2012, 286; Zimmermann, JBl 2012, 20; Ackermann (o. Fn. 5), S. 65 f.; Grigoleit (o. Fn. 5), S. $70 \mathrm{ff}$.

29 Statt aller Roth/Schubert, in: MünchKomm-BGB, 6. Aufl. 2012, 242 Rn. 76 f.

30 Zur Wurzel des $\$ 241$ II BGB im Gebot von Treu und Glauben vgl. nur Roth/Schubert, in: MünchKomm-BGB (o. Fn. 27), \$242 Rn. 170.

31 Vgl. nur Ernst, in: MünchKomm-BGB, 6. Aufl. 2012, \$280 Rn. 93 ff.

32 So etwa Ferrari, in: Schlechtriem/Schwenzer (o. Fn. 10), Art. 7 Rn. 25 ff.; a.A. etwa Viscasillas, in: Kröll/Mistelis/Viscasillas, CISG, 2011, Art. 7 Rn. 24 ff.; jeweils mit zahlreichen weiteren Nachweisen.

33 Staudinger/Magnus (o. Fn. 19), Art. 35 Rn. 35; Müller-Chen, in: Schlechtriem/Schwenzer (o. Fn. 10), Art. 45 Rn. 3; Ziegler, Leistungsstörungsrecht nach dem UN-Kaufrecht, 1995, S. $120 \mathrm{ff}$.

$34 \mathrm{Zu}$ den möglichen Rechtsfolgen eines Verstoßes gegen Treu und Glauben im deutschen Recht vgl. Roth/Schubert, in: MünchKomm-BGB (o. Fn. 29), $\$ 242 \mathrm{Rn} .76 \mathrm{ff}$.

35 Schlechtriem/Schroeter, in: Schlechtriem/Schwenzer (o. Fn. 10), Art. 25 Rn. 7b. 
den, dass Art. 2(2) DCESL hier einen erheblichen Auslegungsspielraum offenlässt, indem er nur ausspricht, dass ein Verstoß gegen Treu und Glauben zu einer Schadensersatzpflicht führen „kann“. Sehr viel deutlicher kommt der Auslegungsspielraum noch in der englischen Version zum Ausdruck („may make the party liable“ - und nicht „makes the party liable“). Denn in der deutschen Fassung könnte man das „kann“ auch in dem Sinne verstehen, dass der Gläubiger wählen darf, auf welche Rechtsfolge er sich berufen möchte. Zudem werden die beiden Rechtsfolgen des Art. 2(2) DCESL einfach nebeneinander gestellt. Anhaltspunkte dafür, wie der Auslegungsspielraum des Art. 2(2) DCESL auszufüllen ist, finden sich nicht. Damit kann die Gefahr nicht ausgeschlossen werden, dass die Gerichte bei Anwendung des Art. 2(2) DCESL sehr viel schneller bei einer Schadensersatzpflicht sind als etwa nach deutschem Recht oder nach UN-Kaufrecht.

Auch die weiteren Haftungsvoraussetzungen des Art. 2(2) DCESL sind unklar: ${ }^{36}$ Art. 2(2) DCESL verweist anders als etwa Art. 106(1)(e) und Art. 131(1)(d) DCESL nicht auf Kapitel 16: Bedeutet das, dass die Einschränkung des Art. 159(1) DCESL, die Pflichtverletzung dürfe nicht i.S.d. Art. 88 DECSL entschuldigt sein, nicht anwendbar ist? Wenn Art. 2(2) DCESL davon spricht, dass der Schuldner für ,jeden Verlust“ haftbar gemacht werden kann, bedeutet dies, dass es auf die Vorhersehbarkeit i.S.d. Art. 161 DCESL wie auch auf die weiteren Einschränkungen des Kapitel 16 nicht ankommt? Auf die Frage nach der Anwendbarkeit des Haftungsausschluss bei Entschuldbarkeit nach Artt. 159(1), 88 DCESL auf die Schadensersatzhaftung aus Art. 2(2) DCESL werde ich unten noch zurückkommen. ${ }^{37}$

Im Ergebnis müssen also beide Parteien fürchten, dass Art. 2 (2) DCESL zu einer erheblich weiteren Haftung führt, als dies nach UN-Kaufrecht und etwa nach deutschem Recht der Fall ist. Sie müssen fürchten, dass jeder Verstoß gegen das Gebot von Treu und Glauben zu einer Schadensersatzhaftung führt, ohne dass die Haftung auf Tatbestandsseite eingeschränkt wird, ohne dass sich der Schuldner auf eine Entschuldbarkeit berufen kann und ohne dass es auf die Vorhersehbarkeit des Schadens ankommt. Das wäre dann hinnehmbar, wenn die Parteien die Haftung privatautonom steuern könnten, aber dem steht Art. 2(3) DCESL entgegen. Art. 2(3) DCESL verbietet es nicht nur, den Pflichtenkanon näher festzulegen, denn dann würden die Parteien in ihrem Vertrag von Art. 2(1) DCESL abweichen und dessen Wirkungen abändern. Sie dürfen ebensowenig einschränkende Haftungsvoraussetzungen vereinbaren. Art. 2(3) DCESL schließt dabei nicht nur gegenüber Verbrauchern jeden privatautonomen Gestaltungsspielraum aus, sondern auch unter Unternehmern.

Führt hier die endgültige Fassung des Gemeinsamen Europäischen Kaufrechts nicht zu mehr Klarheit, werden diese Befürchtungen, selbst wenn sie sich auf Grundlage der sich entwickelnden Rechtsprechung als unbegründet erweisen werden, die Parteien davon abhalten, sich auf das Gemeinsame Europäische Kaufrecht einzulassen: Käufer und Verkäufer sind gleichermaßen größeren und durch privatautonome Vereinbarung nicht kontrollierbaren Haftungsrisiken ausgesetzt. Und auf Akzeptanz bei britischen Marktteilnehmern, die dem Gebot von Treu und Glauben traditionell reserviert gegenüber stehen, wird Art. 2(2) DCESL auch nicht hoffen dürfen. ${ }^{38}$

\section{Die Vertragsauslegung}

Die Regeln zur Vertragsauslegung finden sich im DCESL in den Artt. 58-65. ${ }^{39}$ Art. 58 DCESL enthält drei Auslegungsregeln:

„1. Ein Vertrag wird nach dem gemeinsamen Willen der Parteien ausgelegt, auch wenn dieser nicht mit der normalen Bedeutung der im Vertrag verwendeten Ausdrücke übereinstimmt.

2. Wenn eine Partei einen im Vertrag verwendeten Ausdruck in einem bestimmten Sinne verstanden wissen wollte und dies der anderen Partei bei Vertragsschluss bewusst war oder hätte bewusst sein müssen, wird der Vertrag so ausgelegt, wie die erste Partei ihn verstanden wissen wollte.

3. Sofern die Absätze 1 und 2 nicht anders bestimmen, ist der Vertrag in dem Sinne auszulegen, den ihm eine vernünftige Person geben würde.“

Art. 58 DCESL entspricht im Wesentlichen seiner unmittelbaren Vorgängervorschrift in Art. II.-8:101 DCFR; er findet seine Entsprechung in dem fast wortgleichen Art. 5:101 PECL; in Art. 4.1 UNIDROIT PICC (2010) und Art. 8 CISG finden wir ähnliche Auslegungsregeln; und auch die übrigen Auslegungsregeln des DCESL enthalten nichts, was nicht bereits aus den Vorgängertexten bekannt ist und der Rechtslage in vielen europäischen Rechten entspricht. ${ }^{40}$

Es steht daher nicht zu befürchten, dass die Auslegungsregeln des Gemeinsamen Europäischen Kaufrechts im Vergleich zum UN-Kaufrecht höhere Transaktionskosten verursachen werden. Auch bestehen zumindest aus der Sicht eines kontinentaleuropäischen Juristen keine Bedenken, dass diese Auslegungsregeln auf die Bedürfnisse des grenzüberschreitenden Verkehrs zugeschnitten sind. Und schließlich lässt sich auch keine rechtliche Besserstellung einer der Vertragsparteien durch das Gemeinsame Europäische Kaufrecht im Vergleich zum UN-Kaufrecht feststellen, so dass sich die andere Partei einer Verwendung des Gemeinsamen Europäischen Kaufrechts widersetzen wird.

Aber wie sieht es mit der Akzeptanz durch britische Juristen aus? Hier bestehen erheblich Bedenken. Die Bedenken treten deutlich in den jüngsten Arbeiten der Scottish Law Commission zutage. Im Februar 2010 stellte sie in ihrem Eighth Programme of Law Reform ihr Arbeitsprogramm für die nächsten fünf Jahre vor. ${ }^{41}$ Sie will unter anderem das Vertragsrecht im Lichte des

36 Zum folgenden schon Wendehorst, in: Remien/Herrler/Limmer (o. Fn. 2), S. 193 f.; Koch, in: Wendehorst/Zöchling-Jud (o. Fn. 5), S. 230.

37 Vgl. unten den Text nach Fn. 90.

38 Siehe Law Commission, Scottish Law Commission, An Optional Common European Sales Law: Advantages and Problems. Advice to the UK Government, 2011, $\$ 7.89$.

39 Vgl. hierzu bereits Maultzsch, in: Schmidt-Kessel (o. Fn. 5), S. 203-226; Wendehorst, in: dies./Zöchling-Jud (o. Fn. 5), 87-105; Hardy, ERPL 2011, 817-833.

40 So auch die Einschätzung von Maultzsch (o. Fn. 39), S. 203 ff. Gerade auch im Vergleich zum UN-Kaufrecht bewertet etwa Lando (o. Fn. 2), S. 17, die Auslegungsregeln des Gemeinsamen Europäischen Kaufrechts positiv. Vgl. weiterhin die Auslegungsregeln der einzelnen Vorgängerregeln bewertend Staudinger/Magnus (o. Fn. 19), Art. 8 Rn. 3.

41 Scottish Law Commission, Eighth Programme of Law Reform, Edinburgh 2010 . 
DCFR auf den Prüfstand stellen. ${ }^{42}$ Im Februar 2011 legte sie in einem Discussion Paper zur Vertragsauslegung erste Ergebnisse ihrer Arbeit vor. ${ }^{43}$ Sie kommt darin zu dem vorläufigen Ergebnis, dass sie dem schottischen Gesetzgeber nicht die Übernahme der Vertragsauslegungsregeln des DCFR anempfehlen werde. Sie hat dabei vor allem Bedenken an Art. II.-8:101(1) DCFR, der sich in Art. 58(1) DCESL wiederfindet. Die Scottish Law Commission glaubt, dass der DCFR einem subjektiven Ansatz der Vertragsauslegung folgt. ${ }^{44}$ Ein solcher Ansatz sei zumindest problematisch und werde in Schottland auf Bedenken stoßen: ${ }^{45}$ So berücksichtige er nicht, dass ein Vertrag nicht allein Sache der Vertragsparteien sei. Dritte könnten mit dem Vertrag in Berührung kommen, etwa als Begünstigter aus einem Vertrag zugunsten Dritter oder als Zessionar. Deren Interessen seien durch einen subjektiven Ansatz gefährdet. Zudem generiere, so die Scottish Law Commission, ein subjektiver Ansatz höhere Transaktionskosten. ${ }^{46} \mathrm{Im}$ Ergebnis favorisiert die Scottish Law Commission daher einen objektiven Ansatz der Vertragsauslegung, wie er dem schottischen, aber auch dem englischen Recht entspreche. ${ }^{47}$

Obwohl Art. 58(1) DCESL, Art. 8(1) CISG, Art. II.-8:101(1) DCFR, Art. 5:101(1) PECL und Art. 4.1(1) UNIDROIT PICC (2010) als Auslegungsziel die Ermittlung des gemeinsamen Willens der Parteien festlegen, ist diese Auslegungsregel in der Praxis von nur untergeordneter Bedeutung. ${ }^{48}$ In der Mehrzahl der Fälle wird ein gemeinsamer wirklicher Wille der Parteien nicht ermittelbar sein, so dass es auf die Auslegung aus Sicht eines vernünftigen Dritten ankommt: Art. 58(3) DCESL, Art. 8(2) CISG, Art. II. - 8:101(3) DCFR, Art. 5:101(3) PECL und Art. 4.1(2) UNIDROIT PICC (2010). Damit beruhen die Ausführungen der Scottish Law Commission wohl auf einem Missverständnis. ${ }^{49}$ Doch bringen sie sehr deutlich die Vorbehalte britischer Juristen und Marktteilnehmer gegen die kontinental-europäisch geprägten Auslegungsregeln, die auch das Gemeinsame Europäische Kaufrecht übernimmt, zum Ausdruck. Und diese Vorbehalte werden sich auch auf die Akzeptanz des Gemeinsamen Europäischen Kaufrechts im Vereinigten Königreich auswirken. Diese Vorbehalte ließen sich wohl zumindest zum Teil dadurch einfangen, dass man die Absätze des Art. 58 DCESL einfach anders reiht und mit dem ohnehin in der Praxis im Vordergrund stehenden Grundsatz der objektiven Auslegung beginnt und die weiteren Absätze als Ausnahmen formuliert. ${ }^{50}$

\section{Missbräuchliche Vertragsbestimmungen}

Das achte Kapitel des DCESL zu missbräuchlichen Vertragsklauseln zerfällt in drei Abschnitte. Im ersten Abschnitt finden wir in den Artt. 79-81 DCESL eine Bestimmung zu den Rechtsfolgen der Unwirksamkeit missbräuchlicher Klauseln, eine zu den möglichen Kontrollgegenständen der Inhaltskontrolle und eine Vorschrift, welche die Bestimmungen des achten Kapitels für zwingend erklärt. Der zweite Abschnitt widmet sich in den Artt. 82-85 DCESL der Inhaltskontrolle in Verträgen zwischen einem Unternehmer und einem Verbraucher. Dieser Abschnitt interessiert im Rahmen dieses Beitrages nicht. Der dritte Abschnitt erkennt schließlich in Art. 86 DCESL eine Inhaltskontrolle in Verträgen zwischen Unternehmern an.
Dass der Entwurf des Gemeinsamen Europäischen Kaufrechts überhaupt eine Inhaltskontrolle anerkennt, stellt gegenüber dem UN-Kaufrecht einen Fortschritt dar. Das UN-Kaufrecht weist hier eine sogenannte externe Lücke auf, die unter Rückgriff auf das kollisionsrechtlich einschlägige nationale Recht zu schließen ist. ${ }^{51}$ Marktteilnehmer müssen mitunter damit rechnen, dass ihre Allgemeinen Geschäftsbedingungen einer immer wieder unterschiedlich strengen Inhaltskontrolle ausgesetzt sind, sofern sie sich nicht immer auf dieselbe zu wählende Rechtsordnung mit einem möglicherweise geringen Schutzniveau - einigen. Diese Gefahr birgt das Gemeinsame Europäische Kaufrecht nicht. Auch könnte der deutsche Verwender Allgemeiner Geschäftsbedingungen darauf hoffen, dass die Kontrolle nach dem Gemeinsamen Europäischen Kaufrecht weniger streng ausfällt als die nach deutschem Recht. Die Anwendung der $\$ \$ 307 \mathrm{ff}$. BGB durch deutsche Gerichte auf Allgemeine Geschäftsbedingungen im Unternehmerverkehr wird bereits seit längerem kritisch hinterfragt..$^{52}$ Die Inhaltskontrolle nach Art. 86(1) DCESL ist auf den ersten Blick weniger streng: ${ }^{53}$

„In einem Vertrag zwischen Unternehmern gilt eine Vertragsbestimmung für die Zwecke dieses Abschnitts nur dann als unfair, wenn (a) sie Bestandteil von nicht individuell ausgehandelten Vertragsbestimmungen im Sinne von Artikel 7 ist und (b) so beschaffen ist, dass ihre Verwendung unter Verstoß gegen das Gebot von Treu und Glauben und des redlichen Geschäftsverkehrs gröblich von der guten Handelspraxis abweicht."

Die Auslegung des Art. 86(1) DCESL bereitet freilich Schwierigkeiten, und es überrascht wenig, dass etwa deutsche Juristen mit ihrem national geprägten Vorverständnis an diese Norm heran-

42 Scottish Law Commission, Eighth Programme of Law Reform (o. Fn. 38), $\$ 2.16$.

43 Scottish Law Commission, Review of Contract Law. Discussion Paper on Interpretation of Contract, Discussion Paper No. 147, Edinburgh 2011. Vgl. hierzu Hogg, (2011) 15 Edinburgh LR 406-422; Hellwege, Der DCFR als Grundlage für eine Revision und Kodifikation des schottischen Vertragsrechts?, demnächst in der ZEuP.

44 Scottish Law Commission Discussion Paper No. 147 (o. Fn. 43), $\$ 6.28$

45 Scottish Law Commission Discussion Paper No. 147 (o. Fn. 43), §\$ 6.12 ff., $7.30 \mathrm{ff}$.

46 Vgl. Scottish Law Commission Discussion Paper No. 147 (o. Fn. 43), \$ 6.10.

47 Scottish Law Commission Discussion Paper No. 147 (o. Fn. 43), \$6.29.

48 Wendehorst (o. Fn. 39), S. 88 f.; Vogenauer, in: ders./Kleinheisterkamp (Hrsg.), Commentary on the UNIDROIT Principles of International Commercial Contracts, 2009, Art. 4.1 Rn. 16; Schmidt-Kessel, in: Schlechtriem/Schwenzer (o. Fn. 10), Art. 8 Rn. 10, 19; Kosche, Contra proferentem und das Transparenzgebot im Common Law and Civil Law, 2011, S. 31. Allgemein Zimmermann, in: Festschr. f. Eduard Picker, 2010, S. 1357.

49 Vgl. bereits Hellwege, Der DCFR als Grundlage für eine Revision und Kodifikation des schottischen Vertragsrechts?, demnächst in der ZEuP.

50 Ähnlich, wenn auch mit anderer Begründung, Hardy, ERPL 2011, 817-833.

51 Ferrari, in: Schlechtriem/Schwenzer (o. Fn. 10), Art. 4 Rn. 20; Piltz (o. Fn. 16), Rn. 3-91; Staudinger/Magnus (o. Fn. 19), Art. 4 Rn. 25; Hennemann, AGB-Kontrolle im UN-Kaufrecht aus deutscher und französischer Sicht, 2001, S. 104. Aus der Rechtsprechung OLG Düsseldorf, CISG-online Nr. 915; OGH Wien, CISG-online Nr. 642.

52 Vgl. zu dieser Diskussion etwa Ulmer/Schäfer, in: Ulmer/Brandner/ Hensen, 11. Aufl. 2011, $\$ 310$ Rn. 8; Basedow, in: MünchKomm-BGB, 6. Aufl. 2012, § 310 Rn. $14 \mathrm{f}$.

53 Vgl. auch Ernst (o. Fn. 14), 103; Möslein, in: Schmidt-Kessel (o. Fn. 5), S. 264 . 
treten. So glaubt Friedrich Graf von Westphalen, dass die Kontrolle von Vertragsklauseln nach deutschem AGB-Recht im Ergebnis der von Art. 86(1) DCESL gleicht: ${ }^{54}$ Maßgebliches Bewertungskriterium sei jeweils Treu und Glauben.

Doch bestehen ganz entscheidende Unterschiede. Im deutschen Recht kommt dem dispositiven Recht auch im Unternehmerverkehr eine Leitbildfunktion zu: ${ }^{55} \S 307$ II Nr. 1 BGB. Die erst durch einen Vergleich der Klausel mit dem dispositiven Recht festgestellte unangemessene Benachteiligung muss sodann den Geboten von Treu und Glauben zuwiderlaufen. Bei Verträgen zwischen Unternehmern sind dabei nach $\$ 310$ I 2 Hs. 2 BGB noch zusätzlich „auf die im Handelsverkehr geltenden Gewohnheiten und Gebräuche" Rücksicht zu nehmen. ${ }^{56}$

Für Verbraucherverträge geht der DCFR ebenfalls von einer Leitbildfunktion des dispositiven Rechts aus. Nach Art. II. -9:403 DCFR ist eine nicht individuell ausgehandelte Vertragsklausel missbräuchlich „if it significantly disadvantages the consumer, contrary to good faith and fair dealing“. Die Leitbildfunktion des dispositiven Rechts machen die Autoren des DCFR an der Wendung „disadvantages“ fest. ${ }^{57}$ Eine Benachteiligung des Verbrauchers könne festgestellt werden, wenn die Vertragsklausel mit dem dispositiven Recht verglichen werde. Zwar spricht Art. 83 DCESL nicht mehr von „significantly disadvantages“, sondern in der deutschen Fassung von einem „Ungleichgewicht zu Lasten des Verbrauchers". Doch dürften auch die Autoren des DCESL der Ansicht sein, dass das Ungleichgewicht wiederum durch einen Vergleich der Vertragsklausel mit dem dispositiven Recht festgestellt werden kann. Das Ungleichgewicht muss sodann erheblich sein, und es muss ein Verstoß gegen das Gebot von Treu und Glauben und des redlichen Geschäftsverkehrs vorliegen.

In Art. 86(1) DCESL finden wir auch das Gebot von Treu und Glauben und des redlichen Geschäftsverkehrs. Aber die Voraussetzung des Ungleichgewichts fehlt. ${ }^{58}$ An dessen Stelle steht, dass die Vertragsklausel von der guten Handelspraxis abweichen muss, und diese Abweichung muß gröblich sein. Wenn nun aber die Leitbildfunktion des dispositiven Rechts an dem Begriff „Ungleichgewicht“ festgemacht wird, so lässt die Fassung des Art. 86 (1) DCESL nur einen Schluss zu: Das Gemeinsame Europäische Kaufrecht erkennt dem dispositiven Recht bei der Kontrolle missbräuchlicher Klauseln im Unternehmerverkehr keine Leitbildfunktion zu. Leitbildfunktion hat die gute Handelspraxis! Es genügt dabei nicht jede Abweichung von der guten Handelspraxis. Sie muss gröblich sein, ${ }^{59}$ und es muss darin ein Verstoß gegen das Gebot von Treu und Glauben und des redlichen Geschäftsverkehrs liegen.

Damit weicht Art. 86(1) DCESL geradezu dramatisch von der deutschen Rechtslage ab. Auch Art. 3(3) der Richtlinie 2000/ 35/EG, ${ }^{60}$ der immer wieder als Vorbild des Art. 86(1) DCESL zitiert, ${ }^{61}$ aber auch schon des Art. II.-9:405 DCFR benannt wird, ${ }^{62}$ geht nicht so weit, stellt er doch ebenfalls auf eine grobe Benachteiligung des Gläubigers ab und verlangt sodann, dass diese Benachteiligung gegen die gute Handelspraxis verstoßen müsse. Wir können also festhalten, dass Art. 86(1) DCESL von seiner Grundkonzeption her erheblich vom deutschen Recht abweicht.

Dennoch ist fraglich, ob dieser Aspekt des Gemeinsamen Europäischen Kaufrechts auf Resonanz unter Marktteilnehmern stoßen wird: ${ }^{63}$ Zum einen sind im Vergleich zum deutschen
Recht Kontrollgegenstand der Inhaltskontrolle im Unternehmerverkehr nicht nur Allgemeine Geschäftsbedingungen, sondern alle nicht individuell ausgehandelte Vertragsbestimmungen. ${ }^{64}$ Zum anderen ist es zunächst eine bloße Hoffnung, dass die Inhaltskontrolle nach Art. 86 DCESL weniger streng ausfällt als nach deutschem Recht. ${ }^{65}$ Und schließlich fehlt jede Form von Rechtssicherheit: Die Inhaltskontrolle des Art. 86 DCESL verfolgt mit der Leitbildfunktion der guten Handelspraxis einen neuen Weg. Zwar mag man einwenden, dass jede Generalklausel der Inhaltskontrolle zunächst Rechtsunsicherheit erzeugt, bis sie durch die Rechtsprechung konkretisiert worden ist. Indes bestand mit der Leitbildfunktion des dispositiven Rechts zumindest ein sicherer Vergleichsmaßstab, ein fester Anker. Die gute Handelspraxis ist als Vergleichsmaßstab dagegen wenig greifbar. Die Generalklausel des Art. 86 DCESL besteht damit nur aus Variablen. Der Marktteilnehmer erkauft sich seine Hoffnung auf eine weniger strikte Inhaltskontrolle also durch ein erhöhtes Maß an Rechtsunsicherheit. ${ }^{66}$

Zudem besteht noch eine zweite Gefahr. Die gute Handelspraxis kann lokal unterschiedlich ausfallen, selbst wenn der Zusatz "gut" darauf hinweist, dass nicht rein faktische lokale Usanzen gemeint sind. Zwar kann auch die bloße Berücksichtigung lokal unterschiedlicher Handelsbräuche nach $\$ \$ 307,310$ I 2 Hs. 2 BGB dazu führen, dass die Inhaltskontrolle auf lokale Unterschiede Rücksicht nimmt. Doch wird die Gefahr einer unterschiedlichen Anwendung einer Generalklausel erhöht, wenn die gute Handelspraxis sogar Leitbildfunktion hat. Das eigentlich intendierte Ziel, Transaktionskosten zu senken, wird so gefährdet.

Darüber hinaus wirft die Generalklausel des Art. 86 DCESL weitere Fragen auf: Im deutschen Recht hat das dispositive Recht Leitbildfunktion. Der Gerechtigkeitsgehalt des dispositiven Rechts beschränkt insoweit die Privatautonomie. Der Verweis auf „die im Handelsverkehr geltenden Gewohnheiten und Gebräuche“ in $\$ 310$ I 2 Hs. 2 BGB führt der Sache nach zu einer Erweiterung der Privatautonomie: eine Klausel, die in Verbraucherverträgen unwirksam ist, kann wirksam sein. ${ }^{67}$ Nach Art. 86 DCESL führt der gute Handelsbrauch nicht zu einer Erweiterung

54 Graf von Westphalen, NJOZ 2012, 444 ff.; ders., ZIP 2011, $1992 \mathrm{f}$.

55 Hierzu Fuchs, in: Ulmer/Brandner/Hensen (o. Fn. 52), \$307 Rn. 193 ff.

56 Hierzu Wolf, in: ders./Lindacher/Pfeiffer, 5. Aufl. 2009, \$310 Rn. 24 f.

57 von Bar/Clive (Hg.), Principles, Definitions and Model Rules of European Private Law. Draft Common Frame of Reference, Bd. I, 2009, Art II.9:403, Comment B, S. 635.

58 A.A. Pfeiffer, ERPL 2011, 844.

59 Kritisch hierzu Eidenmüller/Jansen/Kieninger/Wagner/Zimmermann, JZ 2012, $279 \mathrm{f}$

60 Richtlinie 2000/35/EG des Europäischen Parlaments und des Rates vom 29.6.2000 zur Bekämpfung von Zahlungsverzug im Geschäftsverkehr. Nunmehr Art. 7(1) der Richtlinie 2011/7/EU des Europäischen Parlaments und des Rates vom 16.2.2011 zur Bekämpfung von Zahlungsverzug im Geschäftsverkehr.

61 So etwa von Westphalen, ZIP 2011, 1991.

62 Pfeiffer, in: Gedächtnisschr. F. Manfred Wolf, 2011, S. 115 ff.; ders., ERPL 2011, $841 \mathrm{f}$.

63 Kritisch auch Ernst (o. Fn. 14), S. 103

64 Kritisch auch Ernst (o. Fn. 14), S. 104.

65 Kritisch, ob sich diese Hoffnung erfüllen wird, auch Ernst (o. Fn. 14), S. 103.

66 So auch die Kritik von Ernst (o. Fn. 14), S. 104.

67 Zur Korrektivfunktion der Handelsbräuche im Rahmen der Inhaltskontrolle vgl. nur Basedow, in: MünchKomm-BGB (o. Fn. 52), § 310 Rn. 9. 
der Inhaltsfreiheit, sondern er setzt ihr unmittelbar Grenzen. Die Rechtfertigung dafür liegt nicht unmittelbar auf der Hand. Wieso soll Handelsbräuchen eine solch weitgehende normative Funktion zukommen? Richtig scheint mir, dass nicht jedem faktischen Handelsbrauch eine Leitbildfunktion zugestanden werden kann. Es muss etwas hinzutreten. Die Leitbildfunktion des dispositiven Rechts erklärt sich aus dem ihm innewohnenden Gerechtigkeitsgehalt. Dass nicht jeder Handelsbrauch genügt, macht auch das Adjektiv "gut" deutlich. Aber was zählt als guter Handelsbrauch? Nur ein solcher, der nicht auf bloßen Zweckmäßigkeitserwägungen beruht, sondern von dem die beteiligten Handelskreise davon ausgehen, dass ihm ein Gerechtigkeitsgehalt immanent ist? Damit sind wir bei der Frage, was die Entstehungsvoraussetzungen eines guten Handelsbrauchs i.S.d. Art. 86 DCESL sind. Entgegen Wolfgang Ernst müssen meines Erachtens die Gebräuche i.S.d. Art. 67(2) nicht mit den guten Handelsbräuchen i.S.d. Art. 86 DCESL identisch sein, eben weil die guten Handelsbräuche i.S.d. Art. 86 DCESL Leitbildfunktion haben. ${ }^{68}$ Was ist zudem der Maßstab der Inhaltskontrolle des Art. 86 DCESL, wenn sich herausstellt, dass die faktisch bestehenden Handelsbräuche nicht gut sind? Stellen wir dann auf gute hypothetische Handelsbräuche ab? Weiterhin frage ich mich, ob Art. 86 DCESL nicht zu einer doppelten Inhaltskontrolle zwingt: In einem ersten Schritt werden faktische Handelsbräuche ermittelt und einer Inhaltskontrolle unterworfen: es muss sich eben um gute Handelsbräuche handeln. In einem zweiten Schritt werden dann die Klauseln an den guten Handelsbräuchen gemessen. Aber was ist der Maßstab für den ersten Schritt: Am Ende doch das dispositive Recht?

Schließlich enthält auch das achte Kapitel die bereits an anderer Stelle festgestellte Regelungslücke: Für Verträge zwischen einem Unternehmer als Verkäufer und einer Person, die weder Verbraucher noch Unternehmer ist, als Käufer ist keine Inhaltskontrolle vorgesehen, obwohl solche Verträge vom Geltungsbereich des Gemeinsamen Europäischen Kaufrechts erfasst sind. ${ }^{69}$ Sollte diese Regelungslücke nicht noch durch den Verordnungsgeber geschlossen werden, so stellt sich auch hier die Frage, wie diese Lücke geschlossen werden kann, durch eine Analogie zu den Artt. 82-85 DCESL oder zu Art. 86 DCELS. Unternehmer sollten bis zur Schließung dieser Lücke durch die Rechtsprechung sicher gehen und für Verträge mit Personen, die weder Verbraucher noch Unternehmer sind, solche AGB gebrauchen, die sie auch im Verkehr gegenüber Verbrauchern verwenden, eine Praxis, die sich transaktionskostensteigernd auswirken wird.

\section{Vorvertragliche Informationspflichten}

Das zweite Kapitel des Gemeinsamen Europäischen Kaufrechts ist vorvertraglichen Informationspflichten gewidmet. Es zerfällt in fünf Abschnitte. Der erste Abschnitt ist den vorvertraglichen Informationspflichten des Unternehmers im Verhältnis zu Verbrauchern gewidmet. ${ }^{70} \mathrm{Er}$ interessiert in diesem Beitrag nicht. Doch ist ein Unternehmer nicht nur in Verträgen mit Verbrauchern Informationspflichten ausgesetzt, sondern auch, wenn die andere Vertragspartei selbst Unternehmer ist. ${ }^{71}$ Der entsprechende Art. 23 DCESL findet sich im zweiten Abschnitt des zweiten Kapitels:
„1. Vor Abschluss eines Vertrags zwischen Unternehmern [...] ist der Verkäufer [...] verpflichtet, dem anderen Unternehmer gegenüber auf jede nach den Umständen geeignete Weise alle Informationen in Bezug auf die wesentlichen Merkmale der zu liefernden Waren [...] offen zu legen, über die er verfügt oder verfügen müsste und deren Nichtoffenlegung gegenüber der anderen Partei gegen das Gebot von Treu und Glauben und den Grundsatz des redlichen Geschäftsverkehrs verstoßen würde.

2. Bei der Prüfung, ob Absatz 1 verlangt, dass der Verkäufer [...] bestimmte Informationen offen legt, sind sämtliche Umstände zu berücksichtigen, insbesondere, (a) ob der Verkäufer [...] über besondere Sachkunde verfügte, (b) die Aufwendungen des Verkäufers [...] für die Erlangung der einschlägigen Informationen, (c) ob der andere Unternehmer die Informationen leicht auf andere Weise hätte erlangen können, (d) die Art der Informationen, (e) die wahrscheinliche Bedeutung der Informationen für den anderen Unternehmer und (f) die gute Handelspraxis in der betreffenden Situation."

Eine solche Informationspflicht im Unternehmerverkehr kennt das UN-Kaufrecht nicht. Auch dem deutschen Recht ist sie fremd. Ihre Wurzeln offenbaren sich, wenn man die rechtsvergleichenden Notes zu Art. II.-3:101 DCFR liest, auf dem Art. 23 DCESL basiert: ${ }^{72}$ Die Autoren zitieren Art. 2 der Verbrauchsgüterkaufrichtlinie ${ }^{73}$ und Art. 35 CISG. Beide Bestimmungen statuieren aber gerade keine Informationspflichten, sondern enthalten die Mangeldefinitionen und entsprechen damit in etwa $\$ 434$ BGB. Indes glauben die Autoren des DCFR, dass diesen Normen auch die Idee einer Informationspflicht zugrundeliege. Sie schreiben: ${ }^{74}$

"It is therefore possible to make explicit that Directive 1999/44/EC art. 2 appears to be based on a disclosure rule: if the goods do not fulfil the requirements of the 'fit for normal purpose test' [...] the seller is obliged to inform the consumer thereof. [...] Therefore it seems quite natural to speak about an indirect information requirement following from the provisions on conformity in the Consumer Sales Directive."

Die Wurzel der in Art. 23 DCESL anerkannten Informationspflichten deuten darauf hin, dass der Verkäufer insbesondere über Mängel informieren muss. Als Rechtsfolge eines Verstoßes gegen eine Informationspflicht bestimmt sodann Art. 29(1) DCESL:

„Eine Partei, die eine sich aus diesem Kapitel ergebende Pflicht nicht erfüllt, haftet für jeden Verlust, der der anderen Partei durch diese Pflichtverletzung entsteht.“

68 Vgl. Ernst (o. Fn. 14), S. 103.

69 Siehe oben den Text zu und nach Fn. 18.

70 Hierzu Looschelders, in: Remien/Herrler/Limmer (o. Fn. 2), S. 109 ff.

1 Hierzu schon Looschelders (o. Fn. 70), S. 109 ff.

von Bar/Clive (o. Fn. 57), Art. II.-3:101, Notes 1-3, S. 203.

73 Richtlinie 1999/44/EG des Europäischen Parlaments und des Rates vom 25.5.1999 zu bestimmten Aspekten des Verbrauchsgüterkaufs und der Garantien für Verbrauchsgüter.

74 von Bar/Clive (o. Fn. 57), Art. II.-3:101, Note 2, S. 203. 
In welchem Verhältnis steht diese Schadensersatzpflicht zu der wegen Lieferung nicht vertragsgemäßer Waren nach Artt. 106(1) (e), 159 ff. DCESL ${ }^{75}$ Die Schadensersatzpflicht nach Artt. 106(1) (e), 159 ff. DCESL kennt zahlreiche Feinheiten: Zunächst ist die Haftung wegen der Lieferung nicht vertragsgemäßer Waren bei einem Vertrag unter Unternehmern nach Art. 104 DCESL ausgeschlossen, „wenn der Käufer zum Zeitpunkt des Vertragsschlusses die Vertragswidrigkeit kannte oder hätte kennen müssen " ${ }^{76}$ Die Informationspflicht nach Art. 23 DCESL ist nicht ausgeschlossen, wenn der Gläubiger die richtige Information auch ohne den Schuldner hätte erlangen können, sondern dies ist nach Art. 23(2)(c) DCESL nur ein zu berücksichtigender Umstand. Nach Art. 106(2)(a) DCESL ist bei einem Vertrag unter Unternehmern Voraussetzung des Schadensersatzanspruchs, dass der Verkäufer die Möglichkeit der Heilung nach Art. 109 DCESL bekommt. Der Schadensersatzanspruch aus Art. 29 DCESL kennt keine vergleichbare Einschränkung. Nach Art. 106(2)(b) DCESL muss bei einem Vertrag unter Unternehmern der Käufer die Vertragsgemäßheit der Waren prüfen und Abweichungen mitteilen, andernfalls verliert er seine Rechte aus Art. 106 DCESL. Der Schadensersatzanspruch aus Art. 29 DCESL kennt keine entsprechende Prüfungs- und Mitteilungsobliegenheit. Nach Art. 106(4) DCESL ist der Schadensersatzanspruch bei Entschuldbarkeit der Nichterfüllung i.S.d. Art. 88 DCESL ausgeschlossen, Art. 29 DCESL kennt keine vergleichbare Einschränkung. ${ }^{77}$ Auf das Problem um die Anwendbarkeit der Entschuldbarkeitsvoraussetzung auf Ansprüche aus Art. 29 DCESL wird unten noch einmal zurückzukommen sein. ${ }^{78}$ Schließlich ist nach Art. 161 DCESL nur der vorhersehbare Schaden ersetzbar, nach Art. 29 DCESL haftet der Schuldner dagegen „für jeden Verlust“ ${ }^{79}$

Doch ist nicht das Problem, dass das DCESL überhaupt Informationspflichten annimmt und bei ihrer Verletzung eine Schadensersatzpflicht anordnet. Denn auch dem UN-Kaufrecht sind Aufklärungspflichten als Nebenpflichten, deren Verletzung zu einer Schadensersatzpflicht führen kann, nicht unbekannt. ${ }^{80}$ Gleiches gilt für das deutsche Recht. ${ }^{81}$ Das Problem ist, dass die Schadensersatzpflicht viel zu weit formuliert wird und damit nicht mehr mit anderen Regelungsmaterien im Einklang steht.

Die gerade aufgezeigten Abstimmungsprobleme lassen sich auch nicht einfach dadurch lösen, dass man die Schadensersatzhaftung aus Art. 29 DCESL hinter der wegen Nichterfüllung zurücktreten lässt, sobald letztere tatbestandlich einschlägig ist. ${ }^{82}$ Auch dann bliebe nicht erklärbar, warum die Haftung bei und ab Eingreifen des Leistungsstörungsrechts nur noch eingeschränkt, im übrigen aber unbeschränkt sein soll.

Sollte der Verordnungsvorschlag in seiner jetzigen Fassung umgesetzt werden, so bleibt abzuwarten, wie die Rechtsprechung auf diese Abstimmungsprobleme reagieren wird. Verkäufer werden wegen dieser sehr weitgehenden Haftung, der sie sich aussetzen werden, kaum einen Anreiz verspüren, einer Verwendung des Gemeinsamen Europäischen Kaufrechts zuzustimmen. Auch ist unwahrscheinlich, dass britische Marktteilnehmer einem Regelwerk zustimmen werden, das eine solch weitgehende, dem englischen Recht unbekannte Informationspflicht statuiert. ${ }^{83}$

Schließlich fällt noch folgendes auf: Nur in zwei Fällen kennt das Gemeinsame Europäische Kaufrecht Informationspflichten, nämlich erstens in Verträgen zwischen einem Unternehmer und einem Verbraucher und zweitens in Verträgen zwischen zwei
Unternehmern. Aber wie sieht es mit Verträgen zwischen einem Unternehmer und einer Person, die weder Unternehmer noch Verbraucher ist, aus? Auch für solche Verträge ist das Kaufrecht verwendbar ${ }^{84}$ Die identische Regelungslücke hatten wir oben bereits in Art. 4 DCESL $^{85}$ und bei der Inhaltskontrolle missbräuchlicher Vertragsklauseln feststellen können. ${ }^{86}$ Der Regelungsgeber hat diese Situation offensichtlich gleich mehrfach übersehen. Der Rechtsanwender muss diese Lücke, soweit sie sich auch noch im endgültigen Gemeinsamen Europäischen Kaufrecht finden wird, schließen. Ihm bleiben zwei Möglichkeiten: Er zieht erstens eine Analogie zu Artt. 13-22 DCESL. Das würde aber dazu führen, dass dem Unternehmer höhere Transaktionskosten entstehen, als wenn er zum UN-Kaufrecht kontrahiert. Denn im Rahmen des UN-Kaufrechts muss der Verkäufer gegenüber Personen, die weder Unternehmer noch Verbraucher sind, keine ähnlich weit gefassten Informationspflichten einhalten. Oder der Rechtsanwender zieht zweitens eine Analogie zu Art. 23 DCESL. Auch diese Lösung befriedigt nicht vollends, könnte man einen Idealverein doch durchaus als schutzwürdiger erachten als Unternehmer. Bis die Rechtsprechung eine Lösung gefunden hat, sollte der Verkäufer den sicheren Weg gehen und auch Personen gegenüber, die weder Unternehmer noch Verbraucher sind, die Informationspflichten der Artt. 13-22 DCESL einhalten. Die dadurch generierten Transaktionskosten werden den Erfolg des Gemeinsamen Europäischen Kaufrechts einschränken.

\section{Das Leistungsstörungsrecht}

Ein Vergleich zwischen dem UN-Kaufrecht und dem Gemeinsamen Europäischen Kaufrecht wäre ohne Einbeziehung des Leistungsstörungsrechts unvollständig, bildet es doch einen Schwerpunkt beider Regelwerke. Freilich ergibt ein erster Überblick, dass sich beide Regelwerke ähneln. ${ }^{87}$ Die bereits formulierte Grundsatzkritik bezieht sich vor allem auf Verbraucherkauf-

75 Vgl. hierzu bereits Looschelders (o. Fn. 70), S. 123 ff.; Koch (o. Fn. 36), S. 230 .

76 Vgl. zu Art. 104 DCESL Faust, in: Remien/Herrler/Limmer (o. Fn. 2), S. $179 \mathrm{ff}$.

$77 \mathrm{Zu}$ diesem Punkt vgl. bereits Eidenmüller/Jansen/Kieninger/Wagner/ Zimmermann, JZ 2012, 277.

78 Vgl. unten den Text nach Fn. 90.

79 Fragend, was diese Wendung bedeuten soll, auch Benninghoff, in: Schmidt-Kessel (o. Fn. 5), S. 107.

80 Vgl. Müller-Chen, in: Schlechtriem/Schwenzer (o. Fn. 10), Art. 45 Rn. 3; Staudinger/Magnus (o. Fn. 19), Art. 35 Rn. 35; Ziegler (o. Fn. 33), S. 120 ff., 123.

81 Vgl. nur Emmerich, in: MünchKomm-BGB, 6. Aufl. 2012, § 311 Rn. 68 ff.

82 So wohl Looschelders (o. Fn. 70), S. $123 \mathrm{ff}$.

83 Zum englischen Recht vgl. nur die Darstellung bei Peel, Treitel. The Law of Contract, 13. Aufl. 2011, $\$ \$ 9-127$ mit zahlreichen Nachweisen aus der Rechtsprechung sowie eine Diskussion der Ausnahmen.

84 Siehe oben den Text zu und nach Fn. 18.

85 Siehe meinen Beitrag in der IHR 5/2012 unter Gliederungspunkt IV.

86 Siehe oben den Text zu und nach Fn. 69.

87 So auch Faust (o. Fn. 76), S. 186. Zu einem Vergleich zwischen UN-Kaufrecht und DCFR vgl. Huber (o. Fn. 4), S. 807-826. Lando erkennt Fortschritte des Gemeinsamen Europäischen Kaufrechts gegenüber dem UNKaufrecht: Lando (o. Fn. 2), S. 16 ff.; Faust (o. Fn. 76), S. 186, sieht dagegen eine vertane Chance darin, dass der Verordnungsvorschlag die Zweifelsfragen des UN-Kaufrechts nicht gelöst habe. 
verträge. ${ }^{88}$ Daneben werden aber auch zahlreiche Details kritisiert, so etwa der wenig gelungene Katalog des Art. 100 DCESL. ${ }^{89}$ Vor allem die uneingeschränkte Bedeutung von einseitig durch den Käufer formulierten und dem Verkäufer zur Kenntnis gebrachten Verwendungsabsichten nach Art. 100(a) DCESL wird vor allem Vertragsparteien aus dem Vereinigten Königreich von einer Verwendung des Gemeinsamen Europäischen Kaufrechts abhalten.

Diese Punkte möchte ich an dieser Stelle nicht weiter vertiefen, sondern mich einer anderen Frage zuwenden: Marktteilnehmer werden wegen der prima facie bestehenden Ähnlichkeiten mit dem UN-Kaufrecht zunächst davon ausgehen, dass die zum UN-Kaufrecht ergangene Rechtsprechung in den Grenzen des Art. 4(1) DCESL zumindest als Auslegungshilfe des Gemeinsamen Europäischen Kaufrechts dienen kann. Ein solcher Rückgriff würde der Rechtssicherheit dienen.

Doch ist Vorsicht geboten. Art. 106(4) DCESL erkennt an, dass ein Schadensersatzanspruch wegen einer Nichterfüllung durch den Verkäufer dann zu keiner Schadensersatzpflicht des Verkäufers führt, wenn die Nichterfüllung entschuldigt ist. Gleiches gilt nach Art. 131(2) DCESL für den Schadensersatzanspruch des Verkäufers bei einer Nichterfüllung des Käufers. Und Art. 159 (1) DCESL stellt noch einmal allgemein fest, dass Schadensersatz nicht gefordert werden kann, wenn die Nichterfüllung entschuldigt ist. Was eine entschuldigte Nichterfüllung ist, legt sodann Art. 88 DECSL: $:^{90}$

„(1) Die Nichterfüllung einer Verpflichtung durch eine Partei ist entschuldigt, wenn sie auf einem außerhalb des Einflussbereichs dieser Partei liegenden Hindernis beruht und wenn von dieser Partei nicht erwartet werden konnte, das Hindernis zum Zeitpunkt des Vertragsschlusses in Betracht zu ziehen oder das Hindernis oder dessen Folgen zu vermeiden oder zu überwinden.

(2) Besteht das Hindernis nur vorübergehend, so ist die Nichterfüllung für den Zeitraum entschuldigt, in dem das Hindernis besteht. Läuft die Verzögerung jedoch auf eine wesentliche Nichterfüllung hinaus, kann die andere Partei sie als solche behandeln.

(3) Die Partei, die nicht zur Erfüllung in der Lage ist, hat die Pflicht sicherzustellen, dass die andere Partei von dem Hindernis und dessen Auswirkungen auf die Fähigkeit der ersteren Partei zur Erfüllung unverzüglich Kenntnis erhält, nachdem die erstere Partei diese Umstände erkannt hat oder hätte erkennen müssen. Die andere Partei hat Anspruch auf Schadensersatz für alle Verluste, die sich aus einer Verletzung dieser Pflicht ergeben."

Auch das UN-Kaufrecht kennt einen Ausschluss eines Schadensersatzanspruches. Nach Art. 79(1) CISG hat eine Partei „für die Nichterfüllung einer ihrer Pflichten nicht einzustehen, wenn sie beweist, dass die Nichterfüllung auf einem außerhalb ihres Einflussbereichs liegenden Hinderungsgrund beruht und dass von ihr vernünftigerweise nicht erwartet werden konnte, den Hinderungsgrund bei Vertragsabschluss in Betracht zu ziehen oder den Hinderungsgrund oder seine Folgen zu vermeiden oder zu überwinden“. Das UN-Kaufrecht erkennt in Art. 79(1) CISG nicht das Verschuldensprinzip des $\$ 280$ I 2 BGB an. ${ }^{91}$ Hauptanwendungsfall des Art. 79(1) CISG ist vielmehr, dass die Erfül- lung wegen höhere Gewalt ausgeschlossen ist..$^{92}$ Man könnte nun vertreten, dass Artt. 159(1), 88 DCESL entsprechend auszulegen ist, dass das Gemeinsame Europäische Kaufrecht mithin von einer Garantiehaftung mit nur eingeschränkter Entschuldigungsmöglichkeit ausgeht..$^{93}$

Freilich wirft eine solche Auslegung Probleme auf: Da ist etwa die sehr weite Schadensersatzpflicht aus Art. 2(2) DCESL. Der Wortlaut des Art. 2(2) DCESL birgt die Gefahr, dass Gerichte bei Verstoß gegen das Gebot von Treu und Glauben schnell eine Schadensersatzpflicht bejahen. ${ }^{94}$ Zudem ist der Schuldner „für jeden Verlust" haftbar, und damit scheint eine Beschränkung des Schadensersatzanspruchs auf vorhersehbare Schäden durch eine Anwendung des Art. 161 DCESL ausgeschlossen zu sein. Schließlich ist den Parteien nicht möglich, die daraus resultierenden sehr umfassenden Haftungsrisiken privatautonom zu steuern: Art. 2(3) DCESL. Es besteht daher das dringende Bedürfnis, die Haftung aus Art. 2(2) DCESL auf andere Weise einzuschränken: Könnte dies dadurch gelingen, dass man dem Schuldner erlaubt, sich auch in diesen Fällen nach Artt.159(1), 88 DCESL zu entschuldigen, obwohl Art. 2 DCESL anders als Art. 106(1)(e) und Art. 131(1)(d) DCESL gerade nicht auf das Kapitel 16 und damit nicht auf die Artt. 159 ff. DCESL verweist? ${ }^{95}$ Demjenigen, der eine solche Anwendung der Artt. 159(1), 88 DCESL auf die Haftung aus Art. 2(2) DCESL bejaht, stellt sich aber sofort eine Folgefrage: Ist es hier angezeigt vom Grundsatz einer Garantiehaftung mit nur eingeschränkter Entschuldigungsmöglichkeit auszugehen? Für das UN-Kaufrecht wird in der Tat vertreten, dass Art. 79 CISG einheitlich auf die Nichterfüllung aller vertraglichen Pflichten angewandt wird. ${ }^{96}$ Doch sollten Artt. 159(1), 88 DCESL im Fall Art. 2(2) DCESL nicht eher im Sinne eines Verschuldensprinzips ausgelegt werden? ${ }^{97}$ Aber welche Folgen hat das dann für das allgemeine Verständnis der Artt. 159(1),

88 Eidenmüller/Jansen/Kieninger/Wagner/Zimmermann, JZ 2012, $280 \mathrm{ff}$.

89 Vgl. Gsell, in: Schulte-Nölke/Zoll/Jansen/Schulze (o. Fn. 5), S. 231 ff.; Eidenmüller/Jansen/Kieninger/Wagner/Zimmermann, JZ 2012, 280. Zu Art. 100 DCESL ausführlich auch Faust (o. Fn. 76), S. 164 ff.

90 Zu Art. 88 DCESL vgl. Faust (o. Fn. 76), S. 179; ders., in: SchulteNölke/ Zoll/Jansen/Schulze (o. Fn. 5), S. 251-278, 265; Schmidt-Kessel, in: ders. (o. Fn. 5), S. $295 \mathrm{ff}$.

91 Vgl. Schwenzer, in: Schlechtriem/Schwenzer (o. Fn. 10), Art. 79 Rn. 1; Staudinger/Magnus (o. Fn. 19), Art. 79 Rn. 1; Ziegler (o. Fn. 33), S. 217 f.; Offermanns, Methoden der Schadensbemessung in internationalen Regelungswerken, 2011, S. 17 ff.; Heilmann, Mängelgewährleistung im UNKaufrecht, 1994, S. 550; Kranz, Die Schadensersatzpflicht nach den Haager Einheitlichen Kaufgesetzen und dem Wiener UN-Kaufrecht, 1989, S. $190 \mathrm{f}$.

$92 \mathrm{Zu}$ den Einzelheiten Schwenzer, in: Schlechtriem/Schwenzer (o. Fn. 10), Art. 79 Rn. 10 ff.; Schlechtriem, Internationales UN-Kaufrecht, 4. Aufl. 2007, Rn. 289; Staudinger/Magnus (o. Fn. 19), Art. 79 Rn. 7 ff.

93 Vgl. hierzu schon Schmidt-Kessel (o. Fn. 90), S. 295 ff.; Remien, in: Schmidt-Kessel (o. Fn. 5), S. 506; Wilhelm, IHR 2011, 233; Kieninger, in: Schulte-Nölke/Zoll/Jansen/Schulze (o. Fn. 5), S. 209; Schopper, in: Wendehorst/Zöchling-Jud (o. Fn. 5), S. 112 f. A. A. Grädler/Köchel, in: Schmidt-Kessel (o. Fn. 5), S. 358 f.

94 Zum folgenden siehe oben Gliederungspunkt V.

95 Bejahend etwa Wendehorst (o. Fn. 36), S. 193 ff. Gegen eine Anwendung der Vorschriften des Kapitel 16 auf die übrigen Schadensersatzansprüche Koch (o. Fn. 36), S. 230.

96 Schwenzer, in: Schlechtriem/Schwenzer (o. Fn. 10), Art. 79 Rn. 5; Staudinger/Magnus (o. Fn. 19), Art. 79 Rn. 11.

97 Fragend auch Eidenmüller/Jansen/Kieninger/Wagner/Zimmermann, JZ 2012, 282. 
88 DCESL? Ganz ähnliche Fragen ließen sich für die Schadensersatzpflicht aus Art. 29(1) DCESL formulieren. ${ }^{98}$

Wir können festhalten, dass die Erwartungshaltung der Marktteilnehmer, dass das Leistungsstörungsrecht des Gemeinsamen Europäischen Kaufrechts ähnlich angewandt werden wird, wie das des UN-Kaufrechts enttäuscht werden könnte: Die Anerkennung neuer und sehr weitgehender Schadensersatzpflichten im Gemeinsamen Europäischen Kaufrecht kann dazu führen, dass die Entlastungsmöglichkeiten des Schuldners, die sich zunächst am UN-Kaufrecht orientieren, im Gemeinsamen Europäischen Kaufrecht anders und sehr viel großzügiger ausgelegt werden. Sollten Artt. 159(1), 88 DCESL als Folge etwa in der Art ausgelegt werden, dass hier das Verschuldensprinzip anerkannt wird, so mag sich das auch auf die Akzeptanz des Gemeinsamen Europäischen Kaufrechts bei Marktteilnehmern aus Rechtsordnungen negativ auswirken, die das Verschuldensprinzip gerade nicht anerkennen. Allzu große Unterschiede zwischen UN-Kaufrecht und Gemeinsamen Europäischen Kaufrecht sind zudem transaktionskostenrelevant. Wir hatten gesehen, dass es Marktteilnehmern wegen des beschränkten Anwendungsbereichs kaum gelingen wird, bei jedem grenzüberschreitenden Kaufvertrag die Verwendung des Gemeinsamen Europäischen Kaufrechts durchzusetzen. Das UN-Kaufrecht wird insbesondere im Verhältnis zu Vertragspartnern, die ihren gewöhnlichen Aufenthalt nicht in Mitgliedstaaten der Europäischen Union haben, von Bedeutung bleiben. Auch Unternehmer mit gewöhnlichem Aufenthalt in einem Mitgliedstaat werden also weiterhin zum UN-Kaufrecht kontrahieren. Solche Marktteilnehmer werden als Folge AGB vorhalten müssen, die auf die unterschiedlichen rechtlichen Rahmenbedingungen reagieren. Abweichungen des Gemeinsamen Europäischen Kaufrechts zum UN-Kaufrecht sollten also mit Bedacht eingeführt werden und zwar insbesondere dort, wo das UN-Kaufrecht verbesserungswürdig ist. Aber ein solcher Fall scheint mir bei dem gerade beleuchteten Beispiel nicht vorzuliegen.

\section{Zusammenfassung}

In den Fällen, in denen das UN-Kaufrecht anwendbar ist und das Gemeinsame Europäische Kaufrecht verwendbar sein wird, wird sich den Marktteilnehmern nach Inkrafttreten eines Gemeinsamen Europäischen Kaufrechts die Frage stellen, welches Regelwerk sie zur Geltung kommen lassen wollen: Bestehen für sie Anreize, dass Gemeinsame Europäische Kaufrecht vorzuziehen mit der Folge, dass das UN-Kaufrecht zumindest in Europa verdrängt wird? Ich bin skeptisch: ${ }^{99}$ (1) Aus zahlreichen Gründen bestehen Bedenken, dass das Gemeinsame Europäische Kaufrecht in den Fällen, in denen beide Regelwerke anwendbar sind, zu einer Reduzierung von Transaktionskosten führt. (2) Das Gemeinsame Europäische Kaufrecht würde dann zu einem wirklichen Mehrwert führen, wenn es auch bei Marktteilnehmern aus Staaten, die nicht Vertragsstaaten des UN-Kaufrechts sind, auf Akzeptanz stoßen wird. Doch gerade mit Blick auf Marktteilnehmer aus dem Vereinigten Königreich besteht Anlass zur Sorge, dass sie das Gemeinsame Europäische Kaufrecht in der Fassung des vorgelegten Entwurfs ablehnen werden. (3) Doch nicht nur im Vereinigten Königreich wird das Gemeinsame Europäische Kaufrecht auf Akzeptanzprobleme stoßen. So sehen sich die Parteien in Art. 2(2) DCESL und in Art. 29 DCESL neuen, kaum abschätzbaren Haftungsrisiken ausgesetzt. Gerade im grenzüberschreitenden Handelsverkehr legen Marktteilnehmer schließlich Wert auf ein hohes Maß an Privatautonomie und auf Rechtssicherheit. Auch diese Wünsche bedient der Entwurf des Gemeinsamen Europäischen Kaufrechts nicht in genügender Weise. Das alles legt die Forderung nahe, dass das Gemeinsame Europäische Kaufrecht in seiner derzeitigen Fassung nicht in Kraft gesetzt werden sollte. Es ist eine grundlegende und zeitintensive Auseinandersetzung mit dem Entwurf und dessen umfassende Überarbeitung dringend geboten.

98 Mögliche Antworten formuliert Schmidt-Kessel (o. Fn. 90), S. 300 f.

99 Skeptisch auch Lando (o. Fn. 2), S. 18: „CISG, however, has a worldwide extension with 77 Member States, and it may be a ticklish matter if a ,desertion from the CISG' in the intra-EU trade becomes a reality."; Ackermann (o. Fn. 5), S. 51 f.

\section{Entscheidungen}

\section{UN-Kaufrecht (CISG)}

\section{Art. 7, 35, 40, 45, 74, 77, 80 CISG}

1. Um den Anforderungen an den gewöhnlichen Gebrauch im Sinne von Art. 35 Abs. 2 Buchst. a CISG gerecht zu werden, muss sich eine gelieferte Ware für diejenigen Verwendungsmöglichkeiten eignen, die nach ihrer stofflichen und technischen Auslegung und der hieran anknüpfenden Verkehrserwartung nahe liegen. Bleiben die tatsächlich vor- handenen Verwendungsmöglichkeiten dahinter zurück, fehlt der Ware die Eignung zum gewöhnlichen Gebrauch, sofern der Verkäufer die bestehende Einschränkung nicht deutlich macht.

2. Die im UN-Kaufrechtsübereinkommen nicht ausdrücklich geregelte Frage, wie Fallgestaltungen zu behandeln sind, in denen die Vertragsparteien zum entstandenen Schaden unabhängig voneinander durch jeweils eigenständige Pflichtverletzungen beigetragen haben, ist gemäß Art. 7 Abs. 2 CISG durch Rückgriff auf die den Art. 77 und 80 CISG zugrunde liegenden allgemeinen Grundsätze 